\title{
Information retention among attendees at a traditional poster presentation session
}

\author{
Adam K. Saperstein ${ }^{1}$, Robert P. Lennon ${ }^{2}$, Cara Olsen ${ }^{3}$, Luke Womble ${ }^{4}$, Aaron Saguil ${ }^{5}$
}

${ }^{1}$ Department of Family Medicine, Uniformed Services University of the Health Sciences Bethesda, Maryland, ${ }^{2}$ Department of Family Medicine, Naval Hospital Jacksonville, Jacksonville Florida, ${ }^{3}$ Department of Preventive Medicine and Biostatistics, Uniformed Services University of the Health Sciences, Bethesda, Maryland, ${ }^{4}$ Department of Family Medicine, Naval Hospital Camp Pendleton Camp Pendleton, California, ${ }^{5}$ Department of Family Medicine, Uniformed Services University of the Health Sciences, Bethesda, Maryland.

Correspondence:

robert.lennon@med.navy.mil

Tel.: + 904588 2621; Fax.: + 9045427394

Received: 8 June 2016; Accepted: 15 August 2016

Key words: Medical education - Retention Conferences.

Dear Editor,

Medical poster presentations provide an interactive opportunity to share work at conferences with limited podium space (1). There is scarce data evaluating how effectively medical poster presentations facilitate attendees' retention of medical knowledge (2). We performed an IRB exempt pilot study to investigate how well attendees retained information presented in medical posters.

Four poster presenters at the 2013 Society of Teachers of Family Medicine (STFM) Annual Spring Conference were invited to participate in our study. Each presenter identified the central theme and the top two "take home points" for their poster. Conference attendees were invited to participate; those who consented were given a map showing the location of the four posters and asked to view them in the manner they normally view posters.

Three and ninety days after the conference, participants were sent an electronic survey asking about basic demographic information, participants' engagement with the posters, and information retention. We expected learners to retain at least $30 \%$ of information conveyed via a medical poster, based on the National Training Laboratory Institute for Applied Behavioral Science's "Learning Pyramid" (3).

Twenty-six attendees responded at both 3 and 90 days with a mean retention of $14.9 \%$ and $11.3 \%$ respectively. The most common reason reported for attending the poster presentation session was "to learn new information." Information retention was associated with interaction with poster presenters for only 1 of the 4 posters. No associations between gender, age, primary reason for attending the poster presentation session, current position or history of having presented posters in the past and information retention were found. The decline was higher in males and in those further removed from their residency.

Although there are many potential benefits of poster presentations at conferences, 
our study demonstrates that in their current format information retention may not be one of them. Future studies might compare retention between randomly selected posters (like this study) and posters the attendees self-select. Future studies might also evaluate the impact of changing traditional poster presentations to higher engagement modalities, as learner engagement is correlated with increased information retention (4). Specific changes could be to upgrade posters to digital interactive media, flipping the poster session by emailing the posters to attendees in advance of the conference and having poster presenters host round table discussion with interested attendees, or by using moderated poster sessions. These modalities might significantly improve retention without losing the other benefits of poster presentations.

Authors' contributions: Conception and design: AS, RL, CO and AS; Acquisition, analysis and interpretation: AS, RL, CO, LW and AS; Drafting the article: AS, RL, LW and AS; Revising it critically for important in- tellectual content: AS, RL, CO, LW and AS; Approved final version of the manuscript: AS, RL, CO, LW and AS.

Conflict of interest: The authors declare that they have no conflict of interest.

Funding: Intramural Grant from the Uniformed Services University of the Health Sciences.

\section{References}

1. Rowe N. Poster presentations - the "then and now" of a popular medium of scientific communication. FEBS News. 2014;2:9-10.

2. Ilic D, Rowe N. What is the evidence that poster presentations are effective in promoting knowledge transfer? A state of the art review. Health Info Libr J. 2013;30(1):4-12.

3. The Learning Pyramid at NTL Institute for Applied Behavioral Science [cited June 7 2016]. Available from: http://people.okanagan.bc.ca/ ddoige/files/Learning\%20Pyramid.jpg.

4. Kenney JL, Banerjee P. "Would Someone Say Something, Please?" Increasing Student Participation in College Classrooms. Journal on Excellence in College Teaching. 2011;22(4):57-81.

Reprints will not be available from the authors. 